\title{
Design, Synthesis and Antibacterial Evaluation of some new 3,5- Diphenylcyclohex-2-en-1-one Derivatives
}

\author{
Ahmed M. El-Saghier*, Omyma A. Abd Allah and Asmaa M. Kadry \\ Chemistry Department, Faculty of Science, Sohag University, Sohag 82524, Sohag, Egypt \\ *E-mail: el_saghier@yahoo.com
}

\begin{abstract}
The moiety of cyclohexene has been showed highly antibacterial activity. New compounds were synthesized from diphenylcyclohexenone via reaction with a variety of reagents to afford branched chain of 1,3-diphenylcyclohexene or fused heterocyclic systems including pyrazole, chromen and pyrrole derivatives. Cyclic ketoketene $s, s$-acetal can be prepared by reaction with $\mathrm{CS}_{2} / \mathrm{Mel}$ under PTC conditions which in turn used as starting material for the synthesis of pyrazolyl and benzothiszolyl derivatives. Most of new synthesized compounds were characterized by using spectral data and were screened for their antibacterial activity.
\end{abstract}

\section{Indexing terms/Keywords}

Diphenylcyclohexene; Chromen; PTC; Benzothiazole; Antibacterial activity.

\section{Academic Discipline And Sub-Disciplines}

Organic Chemistry

\section{SUBJECT CLASSIFICATION}

Heterocyclic Compounds

\section{TYPE (METHOD/APPROACH)}

Synthesis and Experimental Study

\section{Council for Innovative Research}

Peer Review Research Publishing System

Journal: Journal of Advances in Chemistry

Vol. 6, No. 1

editor@cirworld.com

www.cirworld.com, member.cirworld.com 


\section{INTRODUCTION}

Chalcones belong to a class of $\alpha, \beta$-unsaturated aromatic ketones which occur abundantly in nature, and have drawn much attention because of their benefits to human application [1-3]. Michael addition of ethylacetoacetate to chalcone yields 4,6-diaryl-2-oxo-cyclohex-3-ene-1-carboxylate derivatives, which are efficient synthons for fused isoxazoles, pyrazoles and quinazolins [4,5]. Cyclohexenone derivatives are well known lead molecules for the treatment of inflammation and autoimmune diseases [6]. For example, Methyl substituted $\alpha, \beta$-unsaturated cyclohexenones are an important class of compounds in organic synthesis [7-9], which found to be used as herbicides [10] and are employed in the construction of a wide variety of biologically and medicinally important products [11]. Yathirajan and co-workers have synthesized some new cyclohexenone derivatives, which already reported, the crystal and molecular structure studies of some of the related chalcones and cyclohexenone derivatives [12-17]. The compounds have conventionally been prepared by Knoevenagel condensation reaction of ethyl acetoacetate with suitable aldehyde in presence of a base, followed by the acid hydrolysis of the corresponding diesters to yield the substituted cyclohexenones [18]. Some of the compounds have also been prepared by tandem Michael-Dieckmam decarboxylative annulation reactions [19], Moreover; a chiral primary-secondary diamines catalyst system could also efficiently catalyze the Michael-aldol-dehydration reaction between benzoylacetates and $\alpha, \beta$-unsaturated ketones to provide functionalized chiral cyclohexenones in excellent enantioselectivities [20].

Several reports have pointed out the importance of cyclohexenones for antimicrobial and antitubercular activity [21]. Recently, Homogeneous Pd catalysts have been identified for the conversion of cyclohexenone and tetralone $O$-pivaloyl oximes to the corresponding primary anilines and 1-aminonaphthalenes via inspired method of Semmler-Wolff reaction [22]. Also, the dehydrogenation of cyclohexanones affords cyclohexenones or phenols via removal of 1 or 2 equiv of $\mathrm{H}_{2}$, respectively by using $\mathrm{Pd}(\mathrm{DMSO})_{2}(\mathrm{TFA})_{2}$ which considered unique in its high chemoselectivity for the conversion of cyclohexanones to cyclohexenones, without promoting subsequent dehydrogenation of cyclohexenones to phenols [23]. Keeping in view of the useful findings of cyclic oxoketene s,s-acetal group in continuation of our work on 4,6-diaryl-2-oxocyclohex-3-ene-1-carboxylates [24].

\section{RESULTS AND DISCUSSION}

Ethyl-3,5-diphenyl-2-en-1-one-6-carboxylate (1) was reacted with hydrazine directly to give 2-oxo-4,6-diphenylcyclohex-3-enecarboxylic acid hydrazide (2), while the same reaction was carried in presence of TEA to give 4,6-diphenyl2,3a,4,5-tetrahydroindazol-3-one (3) (scheme 1). Their IR spectrum showed new absorption bands corresponding to $\mathrm{C}=\mathrm{O}$, $\mathrm{NH}$, and $\mathrm{NH}_{2}$ groups and disappeared absorption band for $\mathrm{C}=\mathrm{O}$ ester, where $\mathrm{IR}$ of compound 2 showed new absorption bands at $3421,3350-3231$ and $1708 \mathrm{~cm}^{-1}$ due to $\mathrm{NH}, \mathrm{NH}_{2}$ and $\mathrm{C}=\mathrm{O}$ groups, while IR spectrum of compound 3 showed new absorption bands at $3440,1661 \mathrm{~cm}^{-1}$ corresponding to $\mathrm{NH}$ and $\mathrm{C}=\mathrm{O}$ groups. ${ }^{1} \mathrm{H}-\mathrm{NMR}$ spectra $(\delta, \mathrm{ppm})$ of compound 2 revealed new signals at $\delta 6.39,3.16$ due to $\mathrm{NH}_{2}, \mathrm{NH}$ groups, and the other signals were consistent to the proposed structure. Also the reaction was proved by mass spectra which given the perfect molecular weight $\mathrm{m} / \mathrm{z}=306\left(\mathrm{M}^{+}\right)$. Moreover, ${ }^{1} \mathrm{H}$-NMR spectrum of compound 3 revealed new signals at $\delta 9.68,4.20$ due to $\mathrm{NH}$ (disappeared by $\mathrm{D}_{2} \mathrm{O}$ ) and $\mathrm{CH}-\mathrm{C}=\mathrm{O}$ groups. The ${ }^{13} \mathrm{C}-\mathrm{NMR}$ spectrum showed new signal at $\delta 99.58 \mathrm{ppm}$ due to $-\mathrm{CH}-\mathrm{C}=\mathrm{O}$ group, the other signals were consistent to the proposed structure. On refluxing, compound 1 was allowed to react with malononitrile in presence of catalytic amount of pipredine to afford ethyl-2-dicyanomethylene-4,6-diphenylcyclohex-3-ene-1-carboxylate (4) (scheme 1). Its IR spectrum showed disappearance of absorption band corresponding $\mathrm{C}=\mathrm{O}$ ketone and appearance bands for $\mathrm{CN}$ groups at $2191 \mathrm{~cm}^{-1}$. The ${ }^{1} \mathrm{H}$-NMR spectrum of compound 4 revealed new signal at $\delta 3.83 \mathrm{ppm}$ due to $\mathrm{CH}-\mathrm{CN}$, also signals at $\delta 4.09(q), 1.65(\mathrm{t})$ due to $\mathrm{CH}_{2}$ and $\mathrm{CH}_{3}$ groups. ${ }^{13} \mathrm{C}-\mathrm{NMR}$ spectrum showed new signal at $\delta 59.62,158.70,194.15$ due to $\mathrm{CH}-\mathrm{CN}, \mathrm{CN}, \mathrm{C}=\mathrm{O}$ ester groups, respectively (cf. experimental).

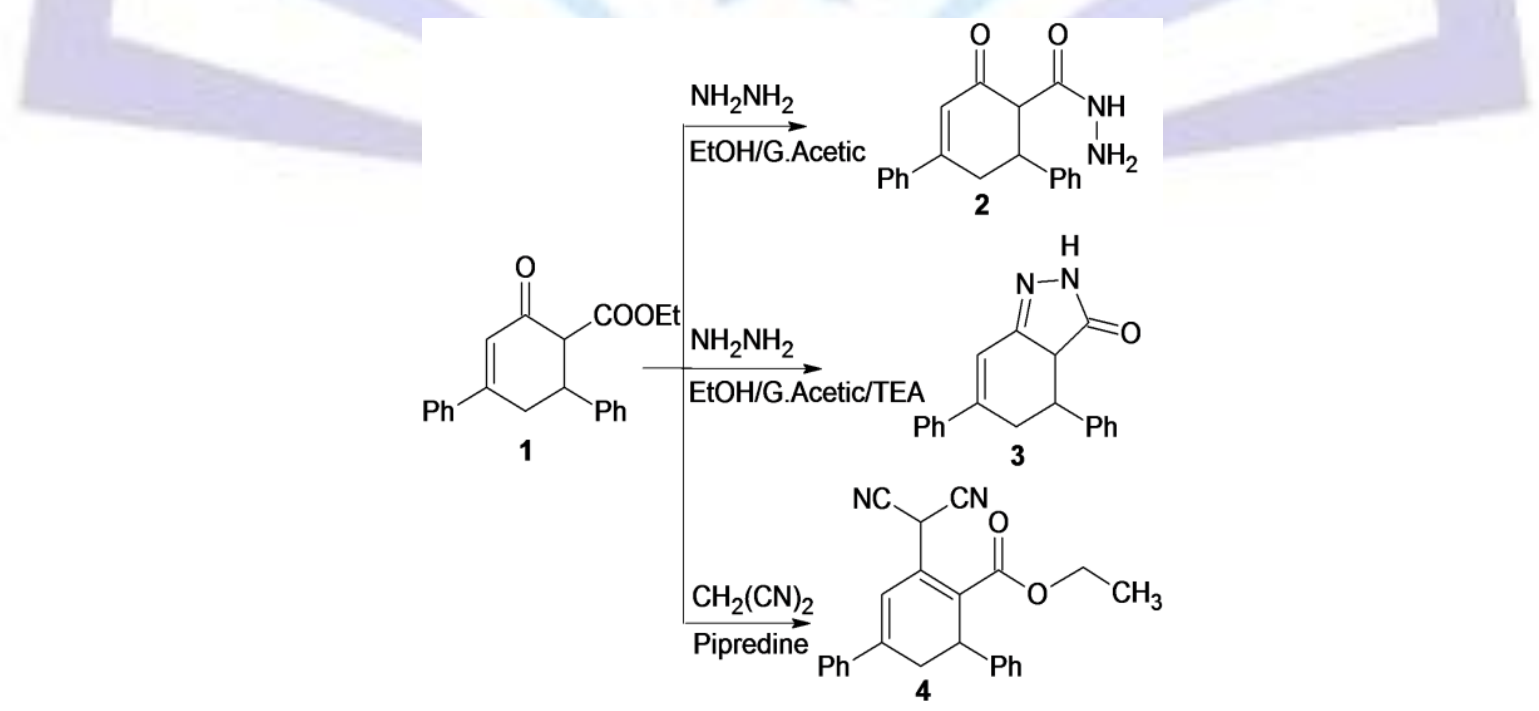

Scheme 1. Reaction of cyclohexenone ester 1 with $\mathrm{NH}_{2} \mathrm{NH}_{2}$ and malononitrile. 
3,5-Diphenylcyclohex-2-enone (5) was synthesized via decarboxylation of compound 1 [4,25] and reacted with $p$ chlorobenzaldehyde to give chalcone derivatives 6 . The ${ }^{1} \mathrm{H}-\mathrm{NMR}$ and ${ }^{13} \mathrm{C}-\mathrm{NMR}$ signals of compound 6 consistent with the proposed structure (cf. experimental). (6E)-6-(4-chlorobenzylidene)-3,5-diphenylcyclohex-2-en-1-one (6) were subjected to react with malononitrile to afford 2-amino-4-(4-chlorobenzylidene-5,7-diphenyl-5,6-dihydro-4H-chromene-3-carbonitrile (7). By another way for the synthesis of compound $\mathbf{7}$ via the reaction of compound $\mathbf{5}$ with $p$-chlorobenzylidene malononitriles (scheme 2.). IR spectrum of compound 7 showed new bands corresponding to $\mathrm{CN}$ and $\mathrm{NH}_{2}$ groups at 2202 and $3434-$ $3321 \mathrm{~cm}^{-1}$, respectively. The ${ }^{1} \mathrm{H}-\mathrm{NMR}$ spectrum showed singlet signals (broad band) at $\delta 2.26 \mathrm{ppm}$ due to $\mathrm{NH}_{2}$ group and disappeared by $\mathrm{D}_{2} \mathrm{O}$, and the other signals consistent with the proposed structure.

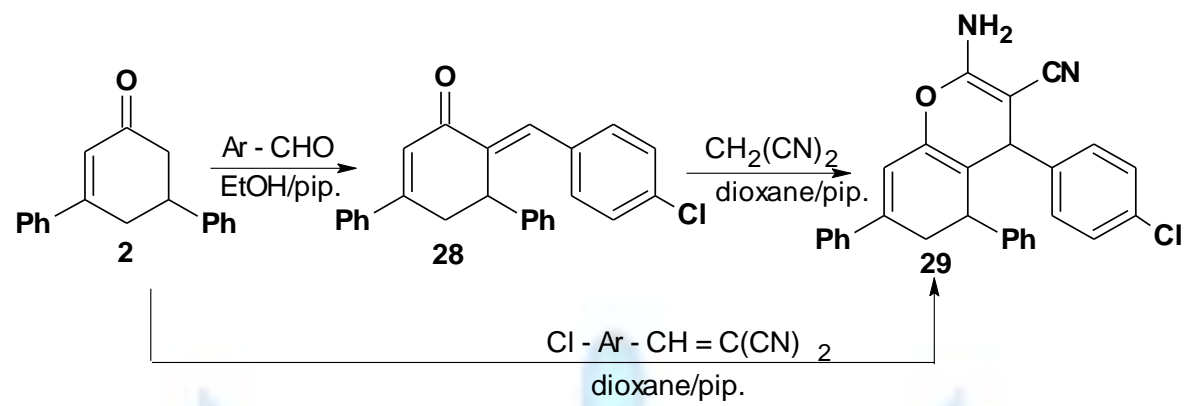

Scheme 2. Synthesis of Chromen derivative.

Ketoketene $S, S$-acetals derivative $\mathbf{8}$ was prepared via the reaction of compound $\mathbf{5}$ with a mixture of carbon disulifide and two moles of methyliodide in one pot. reaction under PTC conditions. Its ${ }^{1} \mathrm{H}-\mathrm{NMR}$ spectrum showed new two singlet signals corresponding to $2 \mathrm{~S}-\mathrm{CH}_{3}$ groups at $\delta 1.58,1.33 \mathrm{ppm}$. The ${ }^{13} \mathrm{C}-\mathrm{NMR}$ signals consistent with the proposed structure (cf. experimental). Moreover, the reaction was proved by mass spectrum which given the molecular ion peak $\mathrm{m} / \mathrm{z}=350$ $\left(\mathrm{M}^{+}-2\right)$.

On the reaction of 6-(bis-methylsulfanylmethylene)-3,5-diphenylcyclohex-2-enone (8) with ethylenediamine or 0 aminothiophenol in ethanol gave the corresponding pyrazolyl and benzthiazolyl derivatives 9 and 10, respectively (scheme 3.). Their ${ }^{1} \mathrm{H}-\mathrm{NMR}$ spectra showed disappearance of signals corresponding to $\mathrm{S}-\mathrm{CH}_{3}$ groups and appeared signals corresponding two methylene group at $\delta 4.15,4.25 \mathrm{ppm}$ in compound 9 and new signal corresponding to $\mathrm{NH}$ group at $\delta$ $2.17 \mathrm{ppm}$ which disappeared by $\mathrm{D}_{2} \mathrm{O}$ (cf. experimental).

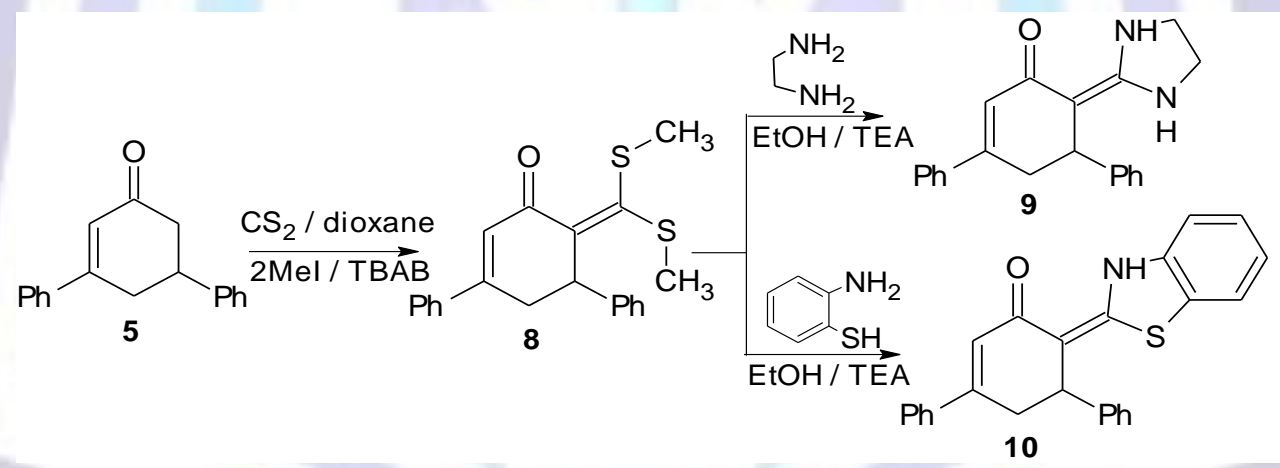

Scheme 3. Using S, S-acetal 8 in synthesis of pyrazolyl 9 and benzothiazolyl derivatives 10.

3,5-Diphenylcyclohex-2-ene hydrazine (11) was synthesized throught the reaction of compound 5 [26] with hydrazine, which reacted with chloroacetyl chloride to give [(2E)-2-(3,5-diphenylcyclohex-2-en-1-ylidene)hydrazino]chloride (12) in excellent yield. Its IR spectrum showed disappearance of absorption bands of $\mathrm{NH}_{2}$ group and appearance of new absorption due to $\mathrm{C}=\mathrm{O}$ and $\mathrm{C}-\mathrm{Cl}$ at $1725,914 \mathrm{~cm}^{-1}$, respectively. ${ }^{1} \mathrm{H}-\mathrm{NMR}$ spectrum showed new signal for $-\mathrm{CH}_{2} \mathrm{Cl}$ group at $\delta 3.12 \mathrm{ppm}$ and the other signals consistent with the proposed structure (cf. experimental). 1,2-diamino-5-oxo-4,5dihydro-1 H-pyrrole-3-carbonitrile (13) was formed through the reaction of compound 12 with malononitrile in DMF (scheme 4.). IR spectrum showed new absorption bands to $\mathrm{C}=\mathrm{O}$ and $\mathrm{NH}_{2}$ groups at 1660 and $3345-3220 \mathrm{~cm}^{-1}$, also new absorption band corresponding to $\mathrm{CN}$ group at $2211 \mathrm{~cm}^{-1}$. The ${ }^{1} \mathrm{H}$-NMR spectrum was consistence with the proposed structure (cf. experimental). 


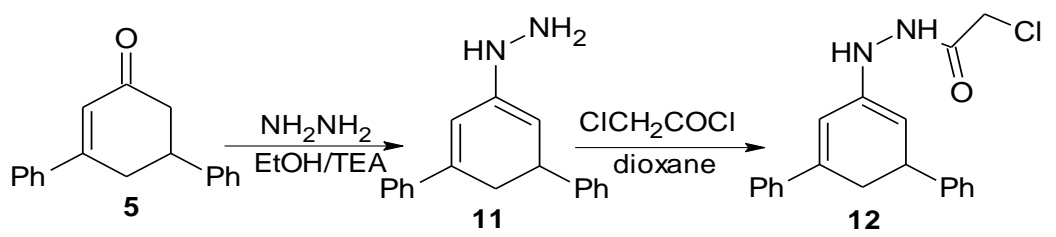

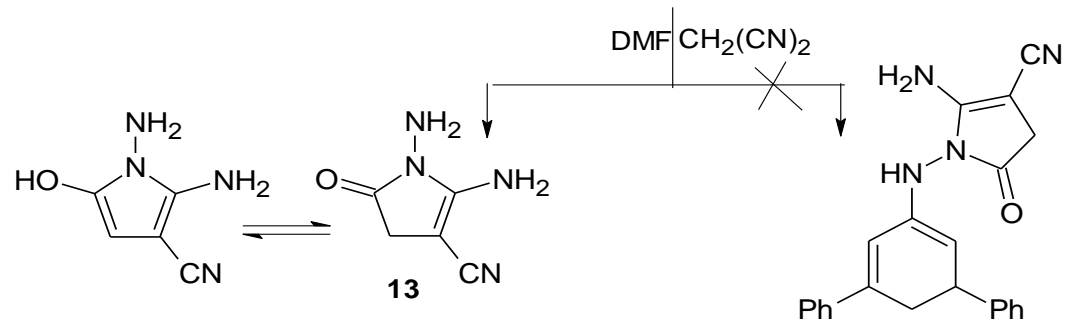

Scheme 4. Reaction of alkylation product of compound 11 with malononitrile.

The mechanism for synthesis of compound $\mathbf{1 3}$ was assumed to proceed via acylation of compound $\mathbf{1 1}$ to afford comound 12 which in turn reacted with malononitrile in presence of DMF with elimination of $\mathrm{HCl}$ molecule followed by nuclophilie attack of $\mathrm{NH}$ group to cyano group to give the cooresponding intermediate pyrrole derivative which was hydrolysed with one mole of $\mathrm{H}_{2} \mathrm{O}$ to give diaminopyrrole 13 (scheme 5).

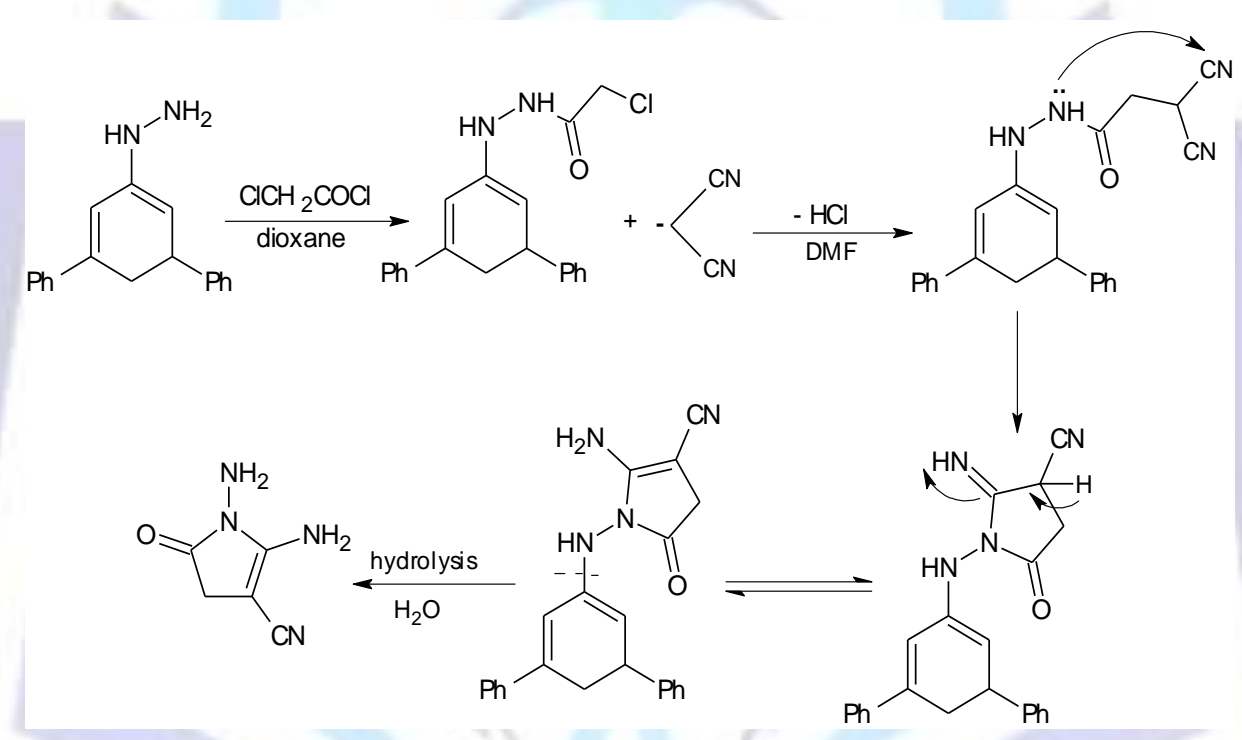

Scheme 5. Expected mechanism of synthesis of pyrrole derivative.

\section{Antibactrial activity evalution}

The newly synthesized heterocyclic compounds 3, 7, 8, 10, 12 and 13 were tested for their inhibitory effect in vitro growth of broad spectrum of bacteria representing one Gram positive bacteria, namely; Bacillus cereus and two Gram negative bacteria, namely; Pseudomonasaeruginosa and Escherichia coli. These compounds exhibited highly antibacterial activity against the tested gram positive, but possess moderate to poor activity against gram negative comparable to chloramphenicol as a standard compound. The results of antibacterial activity are shown in Table 1.

The compounds were dissolved in DMSO. In order to ensure that the solvent had not effect on bacterial growth or enzymatic activity, negative control tests were performed using DMSO at the same concentrations. The inhibitory effect of compounds 3, 7, 8, 10, 12 and 13 in vitro growth of broad spectrum of bacteria was evaluated using agar diffusion method (cup and plate method) [27] by measuring the zone of inhibition on agar plates at three different concentrations 10,000 ppm, 30,000 ppm and 50,000 ppm. DMSO was used as solvent control. All plates were incubated at $37 \pm 0.5 \stackrel{\circ}{\circ}$ for $24 \mathrm{~h}$. The zone of inhibition of compounds was measured using $\mathrm{cm}$ scale. 
Table 1. Antibacterial screening results of the tested compounds

\begin{tabular}{|c|c|c|c|c|c|c|c|c|c|}
\hline $\begin{array}{c}\text { Type of } \\
\text { Bacteria }\end{array}$ & \multicolumn{3}{|c|}{ Bacillus Cereus } & \multicolumn{3}{c|}{ Pseudomonas Aeruginosa } & \multicolumn{3}{c|}{ Escherichia Coli } \\
\hline \multirow{2}{*}{ compound } & \multicolumn{9}{|c|}{ Concentrations in ppm } \\
\cline { 2 - 10 } & 10000 & 30000 & 50000 & 10000 & 30000 & 50000 & 10000 & 30000 & 50000 \\
\hline $\mathbf{3}$ & 1.3 & 1.4 & 1.8 & 1.0 & 1.0 & 1.0 & 1.0 & 1.1 & 1.2 \\
\hline $\mathbf{7}$ & 1.1 & 1.2 & 1.5 & - & - & - & - & - & - \\
\hline $\mathbf{8}$ & 1.8 & 1.8 & 2.0 & 1.2 & 1.2 & 1.3 & 1.2 & 1.2 & 1.2 \\
\hline $\mathbf{1 0}$ & 1.2 & 1.8 & 1.2 & - & - & - & - & - & - \\
\hline $\mathbf{1 2}$ & 1.5 & 1.5 & 1.8 & - & - & - & 1.1 & 1.2 & 1.2 \\
\hline $\mathbf{1 3}$ & 1.3 & 1.5 & 1.5 & - & - & - & 0.9 & 0.9 & 1.0 \\
\hline
\end{tabular}

\section{EXPERIMENTAL PROCEDURE}

Melting points were determined using Kofeler melting point apparatus and were uncorrected. IR $\left(\mathrm{cm}^{-1}\right)$ spectra were recorded as $\mathrm{KBr}$ disc on a Shimadzu DR-8001 spectrophotometer. NMR spectra were carried out at both Micro analytical Unit, Cairo university and NMR lab., chemistry department, Sohag university on a Varian Gemini NMR spectrometer and a Varian Mercury- $300 \mathrm{BB}$ respectively. NMR spectra $400 \mathrm{MHz}$ for ${ }^{1} \mathrm{H}-\mathrm{NMR}$ (DMSO- $d_{6}$ ) and $100 \mathrm{MHz}$ for ${ }^{13} \mathrm{C}-\mathrm{NMR}$ were recorded, the chemical shift is expressed in $\delta$ value $(\mathrm{ppm})$ using TMS as an internal reference. Mass spectra were measured on a VG 7070 E spectrometer (70eV, $200 \mathrm{C}$, direct inlet). Elemental analyses were carried out on a Perkin-Elmer $240^{\circ} \mathrm{C}$ Microanalyzer.

\section{Synthesis of 2-oxo-4,6-diphenyl-cyclohex-3-enecarboxylic acid hydrazide (2).}

To a solution of compound $1(3.21 \mathrm{~g}, 0.01 \mathrm{~mol})$ in ethanol/glacial acetic acid $(1: 1,20 \mathrm{ml})$, hydrazine hydrate $(0.48 \mathrm{ml}$, $0.015 \mathrm{~mol}$ ), was added. The reaction mixture was refluxed for $4 \mathrm{hrs}$. The excess of solvent was evaporated under reduced pressure; the solid obtained was collected and crystallized from ethanol as yellow crystal; yield: $59 \%$; mp 303다. IR $\left(\right.$ KBrymax cm $\left.{ }^{-1}\right)$ : $3421(\mathrm{NH}), 3350-3231\left(\mathrm{NH}_{2}\right), 3049\left(\mathrm{CH}_{\text {arom }}\right), 2925\left(\mathrm{CH}_{\text {aliph. }}\right), 1708(\mathrm{C}=\mathrm{O}), 1650(\mathrm{C}=\mathrm{O}), 1601(\mathrm{C}=\mathrm{N}) ;{ }^{1} \mathrm{H}-\mathrm{NMR}$ (DMSO-d 6 , $\delta$ ppm): 7.51-7.24(m, 10H, CHarom.), 6.59(s, 1H, $\left.\mathrm{CH}_{\text {oliph }}\right), 6.39\left(\mathrm{~s}, 2 \mathrm{H}, \mathrm{NH}_{2}\right.$, disappeared by $\left.\mathrm{D}_{2} \mathrm{O}\right), 3.16(\mathrm{~s}, 1 \mathrm{H}$, $\mathrm{NH}$, disappeared by $\left.\mathrm{D}_{2} \mathrm{O}\right), 2.96(\mathrm{~d}, 1 \mathrm{H}, \mathrm{CH}-\mathrm{C}=\mathrm{O}), 2.72\left(\mathrm{~d}, 2 \mathrm{H}, \mathrm{CH}_{2}\right) 2.38(\mathrm{~m}, 1 \mathrm{H}, \mathrm{CH}-\mathrm{Ph}) ; \mathrm{MS}:(\mathrm{m} / \mathrm{z}, 1 \%): 306.6(14.0)$, 257.8(100), 242(37.2), 199.7(25.6), 116.2(23.3), 94.8(88.4), 56.8(48.8). Anal. Calcd. For $\mathrm{C}_{19} \mathrm{H}_{18} \mathrm{~N}_{2} \mathrm{O}_{2}(306.3):$ C, $74.49 \%$; H, $5.92 \% ;$ N, $9.14 \%$. Found: C, $74.55 \% ; H, 5.96 \% ; N, 9.24 \%$.

\section{Synthesis of 4,6-diphenyl-2,3a,4,5-tetrahydroindazol-3-one (3).}

To a solution of compound $1(3.21 \mathrm{~g}, 0.01 \mathrm{~mol})$ in ethanol/glacial acetic acid $(1: 1,20 \mathrm{ml})$, hydrazine hydrate $(0.48 \mathrm{ml}$, $0.015 \mathrm{~mol}$ ) and a catalytic amount of triethylamine were added. The reaction mixture was refluxed for 4 hrs. The excess of solvent was evaporated under reduced pressure; the solid obtained was collected and crystallized from ethanol as pale yellow crystal; yield: $93 \%$; mp $202^{\circ} \mathrm{C}$, IR (KBrymax cm $\left.{ }^{-1}\right)$ : 3440(NH), 3060 $\left(\mathrm{CH}_{\text {arom. }}\right), 2931\left(\mathrm{CH}_{\text {aliph. }}\right), 1611(\mathrm{C}=\mathrm{O}), 1516(\mathrm{C}=\mathrm{N})$; ${ }^{1} \mathrm{H}-\mathrm{NMR}\left(\mathrm{DMSO}-\mathrm{d}_{6}, \delta \mathrm{ppm}\right): 9.68\left(\mathrm{~s}, 1 \mathrm{H}, \mathrm{NH}\right.$, disappear by $\left.\mathrm{D}_{2} \mathrm{O}\right), 7.11-7.47\left(\mathrm{~m}, 10 \mathrm{H}, \mathrm{CH}_{\text {arom. }}\right), 6.76\left(\mathrm{~s}, 1 \mathrm{H}, \mathrm{CH}_{\text {oliph. }}\right)$, 4.20(d, $1 \mathrm{H}, \mathrm{CH}-\mathrm{C}=\mathrm{O}), 3.18(\mathrm{~m}, 1 \mathrm{H}, \mathrm{CH}-\mathrm{Ph}), 2.93\left(\mathrm{~d}, 2 \mathrm{H}, \mathrm{CH}_{2}\right) ;{ }^{13} \mathrm{C}-\mathrm{NMR}\left(\mathrm{DMSO}-\mathrm{d}_{6}, \delta \mathrm{ppm}\right): 145.75(\mathrm{C}=\mathrm{O}), 140.61(\mathrm{C}=\mathrm{N})$,

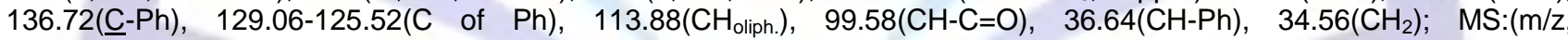
\%):288(100), 271(16.3), 247(27.9), 198(14.0), 128(37.2), 103(34.9), 77(44.2), 59(7.0); Anal. Calcd. For $\mathrm{C}_{19} \mathrm{H}_{19} \mathrm{~N}_{2} \mathrm{O}$ (288.4): C, $79.14 \%$; H, $5.59 \%$; N, $9.72 \%$. Found: C, 79.21\%; H, $5.67 \%$; N, 9.57\%.

\section{Synthesis of ethyl-2-dicyanomethylene-4,6-diphenyl-cyclohex-3-ene-1-carboxylate (4).}

A solution of compound $1(3.21 \mathrm{~g}, 0.01 \mathrm{~mol})$ in $(20 \mathrm{ml})$ ethanol reacted with malonitrile $(0.66 \mathrm{~g}, 0.01 \mathrm{~mol})$, a few drops of pipredine was added. The mixture was heated under reflux for $2 \mathrm{hrs}$, then allowed to cool. The solid product was collected and recrystallized from ethanol as deep Brown crystal; yield: $82 \%$; mp $194{ }^{\circ} \mathrm{C}$, IR $\left(\mathrm{KBrymax} \mathrm{cm}^{-1}\right): 3396(\mathrm{OH})$, 3043 $\left(\mathrm{CH}_{\text {arom }}\right), 2932\left(\mathrm{CH}_{\text {alip }}\right), 2863\left(\mathrm{CH}_{2}\right), 2191(\mathrm{CN}), 1731\left(\mathrm{C}=\mathrm{O}_{\text {ester }}\right), 1656(\mathrm{C}=\mathrm{C}) ;{ }^{1} \mathrm{H}-\mathrm{NMR}\left(\mathrm{DMSO}-\mathrm{d}_{6}, \delta \mathrm{ppm}\right): 7.66-7.29(\mathrm{~m}$, $10 \mathrm{H}, \mathrm{CH}$ of aromatic ring), $6.60\left(\mathrm{~s}, 1 \mathrm{H}, \mathrm{CH}_{\text {oliph. }}\right), 4.09\left(\mathrm{q}, 2 \mathrm{H}, \mathrm{CH}_{2}\right), 3.83(\mathrm{~s}, 1 \mathrm{H}, \mathrm{CH}-\mathrm{CN}), 3.11\left(\mathrm{~d}, 2 \mathrm{H}, \mathrm{CH}_{2}\right), 3.04(\mathrm{~s}, 1 \mathrm{H}, \mathrm{CH}-$ $\mathrm{Ph}), 1.65\left(\mathrm{t}, 3 \mathrm{H}, \mathrm{CH}_{3}\right) ;{ }^{3} \mathrm{C}-\mathrm{NMR}\left(\mathrm{DMSO}-\mathrm{d}_{6}, \delta \mathrm{ppm}\right): 13.97\left(\mathrm{CH}_{3}\right), 36.17\left(\mathrm{CH}_{2}\right), 44.18(\mathrm{CH}-\mathrm{Ph}), 59.62(\mathrm{CH}-\mathrm{CN}), 60.99(\mathrm{CH}$ ester $), \quad 124.08\left(\mathrm{CH}_{\text {oliph }}\right), \quad 126.23-137.70(\mathrm{C}$ of $\mathrm{Ph}), \quad 141.00(\underline{\mathrm{C}}-\mathrm{Ph}), \quad 147.12(\underline{\mathrm{C}}-\mathrm{CH}-\mathrm{CN}), \quad 158.70(\mathrm{CN}), \quad 169.32(\underline{\mathrm{C}}-\mathrm{C}=\mathrm{O})$, 194.15(C=O ester); Anal. Calcd. For $\mathrm{C}_{24} \mathrm{H}_{20} \mathrm{~N}_{2} \mathrm{O}_{2}$ (368.4): C, $78.24 \% ; \mathrm{H}, 5.47 \% ; \mathrm{N}, 7.60 \%$. Found: $\mathrm{C}, 78.25 \% ; \mathrm{H}, 5.55 \%$; $\mathrm{N}, 7.51 \%$.

\section{Synthesis of (6E)-6-(4-chlorobenzylidene)-3,5-diphenyl cyclohex-2-en-1-one (6).}

To a mixture of compound $5(2.48 \mathrm{~g}, 0.01 \mathrm{~mol})$ and $p$-chloro benzaldehyde $(1.18 \mathrm{ml}, 0.01 \mathrm{~mol})$ in ethanol $(30 \mathrm{ml})$, a few drops of piperidine were added. The mixture was heated under reflux for $4 \mathrm{~h}$, then allowed to cool. The solid product was collected and recrystallized from dioxane as white crystal; yield: $67 \%$; mp $186{ }^{\circ} \mathrm{C}$; IR $\left(\mathrm{KBr}\right.$ ymax cm$\left.{ }^{-1}\right): 3040\left(\mathrm{CH}_{\text {arom. }}\right)$, $2986\left(\mathrm{CH}_{\text {aliph }}\right), 1660(\mathrm{C}=\mathrm{O}), 1650(\mathrm{C}=\mathrm{N}), 970(\mathrm{C}-\mathrm{Cl}) ;{ }^{1} \mathrm{H}-\mathrm{NMR}\left(\mathrm{DMSO}-\mathrm{d}_{6}, \delta \mathrm{ppm}\right): 7.92-7.36\left(\mathrm{~m}, 10 \mathrm{H}, \mathrm{CH}_{\text {arom. }}\right), 7.26(\mathrm{~d}, 1 \mathrm{H}$, $=\mathrm{CH}-\mathrm{Ar}-\mathrm{Cl}) 6.45\left(\mathrm{~s}, 1 \mathrm{H}, \mathrm{CH}_{\text {oliph. }}\right), 3.03\left(\mathrm{~d}, 2 \mathrm{H}, \mathrm{CH}_{2}\right), 2.83(\mathrm{t}, 1 \mathrm{H}, \mathrm{CH}-\mathrm{Ph}) ;{ }^{13} \mathrm{C}-\mathrm{NMR}\left(\mathrm{DMSO}-\mathrm{d}_{6}, \delta \mathrm{ppm}\right): 198.8(\mathrm{C}=\mathrm{O})$,

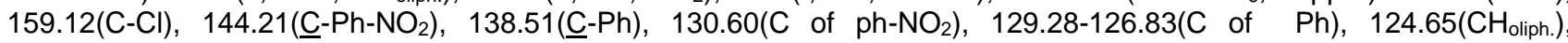


44.04 $(=\underline{\mathrm{CH}}-\mathrm{Ar}-\mathrm{Cl}+\underline{\mathrm{CH}}-\mathrm{ph}), 35.72\left(\mathrm{CH}_{2}\right)$; Anal. Calcd. For $\mathrm{C}_{25} \mathrm{H}_{19} \mathrm{ClO}$ (370.87): $\mathrm{C}, 80.96 \% ; \mathrm{H}, 5.16 \%$; $\mathrm{Cl}, 9.56 \%$. Found: $\mathrm{C}, 80.74 \% ; \mathrm{H}, 5.28 \%$; Cl, $9.66 \%$.

\section{Synthesis of 2-amino-4-(4-chlorobenzylidene-5,7-diphenyl-5,6-dihydro-4H-chromene-3-carbonitrile (7).}

Method 1. A solution of compound $6(3.70 \mathrm{~g}, 0.01 \mathrm{~mol})$ in $(20 \mathrm{ml})$ dioxane reacted with malonitrile $(0.66 \mathrm{~g}, 0.01 \mathrm{~mol})$, a few drops of pipredine was added. The mixture was heated under reflux for $2 \mathrm{hrs}$, then allowed to cool. The solid product was collected and recrystallized from dioxane.

Method 2. A solution of compound $5(2.48 \mathrm{~g}, 0.01 \mathrm{~mol})$ in $(20 \mathrm{ml})$ dioxane reacted with $p$-chlorobenzylidine malonitrile $(1.88 \mathrm{~g}, 0.01 \mathrm{~mol})$, a few drops of pipredine was added. The mixture was heated under reflux for 2 hrs, then allowed to cool. The solid product was collected and recrystallized from dioxane.as pale brown powder; yield: $54 \%$; mp $284 \stackrel{\circ}{\circ}$; IR (ymax cm $\left.{ }^{-1}\right)$ : 3434-3321 $\left(\mathrm{NH}_{2}\right), 3044\left(\mathrm{CH}_{\text {arom. }}\right), 2929\left(\mathrm{CH}_{\text {aliph }}\right), 2202(\mathrm{CN}), 1661(\mathrm{C}=\mathrm{N}), 881(\mathrm{C}-\mathrm{Cl}) ;{ }^{1} \mathrm{H}-\mathrm{NMR}\left(\mathrm{DMSO}-\mathrm{d}_{6}, \delta\right.$ ppm): 8.65-7.23 (m, 14H, CH arom.), $6.89\left(\mathrm{~s}, 1 \mathrm{H}, \mathrm{CH}_{\text {oliph }}\right), 4.77(\mathrm{~s}, 1 \mathrm{H}, \mathrm{CH}-\mathrm{Ar}-\mathrm{Cl}), 3.75\left(\mathrm{~d}, 2 \mathrm{H}, \mathrm{CH}_{2}\right), 3.02(\mathrm{t}, 1 \mathrm{H}, \mathrm{CH}-\mathrm{Ph})$, 2.26(s, $2 \mathrm{H}, \mathrm{NH}_{2}$, disappeared by $\mathrm{D}_{2} \mathrm{O}$ ); Anal. Calcd. For $\mathrm{C}_{22} \mathrm{H}_{18} \mathrm{~N}_{2} \mathrm{O}$ (326.4): C, $80.96 \% ; \mathrm{H}, 5.56 \%$; $\mathrm{N}, 8.58 \%$. Found: C, $80.75 \% ; \mathrm{H}, 5.66 \%$; N, $8.69 \%$.

\section{Synthesis of 6-(bis-methylsulfanyl-methylene)-3,5-diphenyl-cyclohex-2-enone (8).}

To a solution of compound 5 ( $2.48 \mathrm{~g}, 10 \mathrm{mmol})$ in dioxane $(15 \mathrm{ml})$ and catalytic amount of tetrabutyl ammonium bromide, dried potassium carbonate $(3.5 \mathrm{~g}, 0.1 \mathrm{~mol}$ ) was added and the mixture was stirred for $1 \mathrm{hr}$ at room temperature, carbon disulfide $(0.72 \mathrm{ml}, 12 \mathrm{mmol})$ was then added drop wise and the mixture stirred for additional 2 hrs, then iodomethane $(1.24 \mathrm{ml}, 20 \mathrm{mmol})$ was added and the mixture stirred for $4 \mathrm{hrs}$ at r.t. the reaction was quenched with water and the precipitate was filterated off, wash with water, recrystallized from ethanol as pale brown powder; yield: $89 \%$; $\mathrm{mp}$ 79-81 ${ }^{\circ} \mathrm{C}$; IR (KBrymax cm $\left.{ }^{-1}\right): 3057\left(\mathrm{CH}_{\text {aromatic }}\right), 2952\left(\mathrm{CH}_{\text {aliph }}\right), 1656(\mathrm{C}=\mathrm{O}), 1126(\mathrm{C}-\mathrm{S}) ;{ }^{1} \mathrm{H}-\mathrm{NMR}$ (DMSO-d,$\delta$ ppm): : $7.73-$ 7.03(m, $10 \mathrm{H}, \mathrm{CH}$ of aromatic), 6.45(s, $\left.1 \mathrm{H}, \mathrm{CH}_{\text {oliph. }}\right), 3.01\left(\mathrm{~d}, 2 \mathrm{H}, \mathrm{CH}_{2}\right), 2.75(\mathrm{t}, 1 \mathrm{H}, \mathrm{CH}-\mathrm{Ph}), 1.58\left(\mathrm{~s}, 3 \mathrm{H}, \mathrm{CH}_{3}-\mathrm{S}\right), 1.33(\mathrm{~s}, 3 \mathrm{H}$, $\left.\mathrm{CH}_{3}-\mathrm{S}\right) ;{ }^{13} \mathrm{C}-\mathrm{NMR}\left(\mathrm{DMSO}-\mathrm{d}_{6}, \delta \mathrm{ppm}\right): 198.87(\mathrm{C}=\mathrm{O}), 159.16(\mathrm{C}-\mathrm{S}), 144.11(\mathrm{C}=\mathrm{C}), 138.49(\mathrm{C}-\mathrm{Ph}), 130.58(\underline{\mathrm{C}}-\mathrm{Ph}), 129.27-$ 126.76(C of $\left.\mathrm{Ph}), 121.23\left(\mathrm{CH}_{\text {olip. }}\right), 44.04(\underline{\mathrm{CH}}-\mathrm{Ph}), 35.74\left(\mathrm{CH}_{2}\right), 23.56\left(\mathrm{~S}-\mathrm{CH}_{3}\right), 19.65\left(\mathrm{~S}-\underline{\mathrm{CH}}_{3}\right) ; \overline{\mathrm{MS}}\right)(\mathrm{m} / \mathrm{z}, \%): 350,320(0.7)$, 246(3.6), 203(3.2), 144.0(100), 102.2(11.0), 77.0(50.9); Anal. Calcd. For $\mathrm{C}_{21} \mathrm{H}_{20} \mathrm{OS} 2$ (352.5): C, $71.55 \%$; $\mathrm{H}, 5.72 \%$; $18.19 \%$. Found: C, $71.51 \%$; H, $5.56 \%$; S, $18.29 \%$.

\section{Synthesis of 6-imidazolidin-2-yl-3,5-diphenyl-cyclohex-2-enone (9).}

A solution of compound $8(3.52 \mathrm{~g}, 0.01 \mathrm{~mol})$ in $(20 \mathrm{ml})$ ethanol with ethylenediamine $(0.66 \mathrm{ml}, 0.01 \mathrm{~mol})$, catalytic amount of triethyl amine was added. The mixture was heated under reflux for $4 \mathrm{hrs}$, then allowed to cool. The solid product was collected and recrystallized from ethanol as pale yellow powder; Yield: $44 \%$; $\mathrm{mp} 170^{\circ} \mathrm{C}$; IR (KBrymax cm-1): 3420 , 3291(2NH), 3049(CH arom.), 2933(CHaliph), 1668(C=O), 1593(C=N), 1449(C=C)1126 (C-S); 'H-NMR (DMSO-d6, $\delta$ ppm): $7.55-6.98(\mathrm{~m}, 10 \mathrm{H}, \mathrm{CH}$ of aromatic), $6.26(\mathrm{~s}, 1 \mathrm{H}, \mathrm{CHoliph}),. 6.93(\mathrm{~s}, 1 \mathrm{H}, \mathrm{NH}$, disappeared by D2O), 4.42(d, 2H, CH2), 4.25(t, 2H, CH2-NH), 4.15(t, 2H, CH2-NH), 3.87(t, 1H, CH-Ph), 1.23(s, 1H, NH, disappeared by D2O); $\mathrm{MS}:(\mathrm{m} / \mathrm{z}, \mathrm{l} \%)$ : 316.7(7.9) $\left(\mathrm{M}^{+}\right), 245(15.0), 204(11.0), 143.1(13.4), 104.8(100), 76.8(76.4), 54.8(10.2)$; Anal. Calcd. For C21H20N2O (316.4): C, $79.72 \% ; H, 6.37 \%$; N, 8.85. Found: C, 79.51\%; H, 6.47 S, 8.96\%.

\section{Synthesis of 6-(2,3-dihydro-benzothiazol-2-yl)-3,5-diphenyl-cyclohex-2-enone (10).}

A solution of compound $8(3.52 \mathrm{~g}, 0.01 \mathrm{~mol})$ in $(20 \mathrm{ml})$ ethanol with o-amino thiophenol $(1.07 \mathrm{ml}, 0.01 \mathrm{~mol})$, catalytic amount of triethyl amine was added. The mixture was heated under reflux for $4 \mathrm{hrs}$, then allowed to cool. The solid product was collected and recrystallized from ethanol as deep brown powder; Yield: $69 \% ; \mathrm{mp} 138^{\circ} \mathrm{C}$; IR $\left(\mathrm{KBrymax}^{\mathrm{cm}} \mathrm{cm}^{-1}\right)$ : 3445 $(\mathrm{NH}), 3048(\mathrm{CH}$ aromatic $), 2923\left(\mathrm{CH}_{\text {aliph }}\right), 1666(\mathrm{C}=\mathrm{O}), 1605(\mathrm{C}=\mathrm{N}), 1170(\mathrm{C}-\mathrm{S}) ;{ }^{1} \mathrm{H}-\mathrm{NMR}$ (DMSO-d,$\left.\delta \mathrm{ppm}\right):: 7.73-$ $6.61\left(\mathrm{~m}, 15 \mathrm{H}, \mathrm{CH}\right.$ of aromatic), $6.59(\mathrm{~s}, 1 \mathrm{H}, \mathrm{CH}$ arylidine $), 4.25\left(\mathrm{~d}, 2 \mathrm{H}, \mathrm{CH}_{2}\right.$ ring $), 3.74(\mathrm{t}, 1 \mathrm{H}, \mathrm{CH}-\mathrm{Ph}), 2.71(\mathrm{~s}, 1 \mathrm{H}, \mathrm{NH}$, disappeared by $\mathrm{D}_{2} \mathrm{O}$ ); Anal. Calcd. For $\mathrm{C}_{25} \mathrm{H}_{19} \mathrm{NOS}$ (381.4): C, 78.71\%; H, $5.02 \%$; N, $3.67 \%$; S, 8.41 \%. Found: C, 78.60 $\% ; \mathrm{H}, 5.13 \% ; \mathrm{N}, 3.55 \%$;, $8.53 \%$.

\section{Synthesis of (3,5-diphenyl-cyclohexa-1,5-dienyl)-hydrazine (11).}

To a solution of compound $5(2.48 \mathrm{~g}, 0.01 \mathrm{~mol})$ in ethanol $(20 \mathrm{ml})$, hydrazine hydrate $(0.48 \mathrm{ml}, 0.015 \mathrm{~mol})$ and a catalytic amount of triethylamine were added. The reaction mixture was refluxed for $6 \mathrm{~h}$. The excess of solvent was evaporated under reduced pressure; the solid obtained was collected and crystallized from ethanol [literature $156{ }^{\circ} \mathrm{C}(27)$ ] as yellow crystal; yield: $91 \%$; mp $189-190^{\circ} \mathrm{C}$; IR (ymax, $\left.\mathrm{cm}^{-1}\right)$ : 3447-3358( $\left.\mathrm{NH}_{2}\right), 3205(\mathrm{NH}), 3031(\mathrm{CH}$ aromtic.), $2929(\mathrm{CH}$ aliph.), $1645(\mathrm{C}=\mathrm{N}), 1562(\mathrm{C}=\mathrm{C}) ;{ }^{1} \mathrm{H}-\mathrm{NMR}\left(\mathrm{CDCl}_{3}, \delta \mathrm{ppm}\right): 7.41-6.77(\mathrm{~m}, 10 \mathrm{H}, \mathrm{CH}$ aromatic $), 6.60(\mathrm{~s}, 1 \mathrm{H}, \mathrm{CH}$ oliph.), 5.18(s, $2 \mathrm{H}, \mathrm{NH}_{2}$, disappeared by $\left.\mathrm{D}_{2} \mathrm{O}\right), 3.10(\mathrm{~d}, 1 \mathrm{H}, \mathrm{CH}=\mathrm{C}-\mathrm{NH}), 2.77\left(\mathrm{~d}, 2 \mathrm{H}, \mathrm{CH}_{2}\right), 2.22-2.16(\mathrm{~m}, 1 \mathrm{H}, \mathrm{CH}-\mathrm{Ph}), 1.36(\mathrm{~s}, 1 \mathrm{H}, \mathrm{NH}$, disappeared by $\left.\mathrm{D}_{2} \mathrm{O}\right) ;{ }^{13} \mathrm{C}-\mathrm{NMR}\left(\mathrm{CDCl}_{3}, \delta \mathrm{ppm}\right): 29.48(\mathrm{CH}-\mathrm{Ph}), 35.08\left(\mathrm{CH}_{2}\right), 39.80(\mathrm{CH}=\mathrm{C}), 124.46(\mathrm{CH}$ oliph), $124.77-$ 128.81(C of Ph), 140.24(C of PH), $144.80(\mathrm{C}-\mathrm{NH}), 150.25(\mathrm{C}-\mathrm{Ph}) ; \mathrm{MS}:(\mathrm{m} / \mathrm{z}, \%): 262(18.6), 244(100), 227(32.6), 126(30.2)$, 75(18.6), 50(27.9); Anal. Calcd. For $\mathrm{C}_{18} \mathrm{H}_{18} \mathrm{~N}_{2}$ (262): C, $82.41 \% ; \mathrm{H}, 6.92 \% ; \mathrm{N}, 10.68 \%$. Found: $\mathrm{C}, 82.50 \% ; \mathrm{H}, 6.75 \%$; $10.76 \%$.

\section{Synthesis of [(2E)-2-(3,5-diphenylcyclohex-2-en-1-ylidene)hydrazino]chloride (12).}

To a solution of compound $11(2.62 \mathrm{~g}, 0.01 \mathrm{~mol})$ in dry dioxane $(20 \mathrm{ml})$, chloroacetyl chloride $(0.8 \mathrm{ml}, 0.01 \mathrm{~mol})$ and catalytic amount of triethylamine were added.The reaction mixture was refluxed for $6 \mathrm{~h}$. After cooling, the separated solids were collected, crystallized from ethanol as deep yellow crystal; yield: $45 \%$; $\mathrm{mp} 172^{\circ} \mathrm{C}$; IR $\left(\mathrm{ymax}^{-1}\right): 3355(\mathrm{NH})$, 3155(NH), 3040 ( $\left.\mathrm{CH}_{\text {aromatic }}\right), 2928\left(\mathrm{CH}_{\text {aliph }}\right), 1725(\mathrm{C}=\mathrm{O}), 1634(\mathrm{C}=\mathrm{N}), 914(\mathrm{C}-\mathrm{Cl}) ;{ }^{1} \mathrm{H}-\mathrm{NMR}$ (DMSO- $\left.\mathrm{d}_{6}, \delta \mathrm{ppm}\right): 7.47-7.18(\mathrm{~m}$, $\left.10 \mathrm{H}, \mathrm{CH}_{\text {aromatic }}\right), 6.79$ (s, $\left.1 \mathrm{H}, \mathrm{CH}_{\text {oliph. }}\right), 4.15(\mathrm{~s}, 1 \mathrm{H}, \mathrm{NH}), 3.63(\mathrm{~s}, 1 \mathrm{H}, \mathrm{NH}), 3.39$ (d, $\left.1 \mathrm{H}, \mathrm{CH}=\mathrm{C}-\mathrm{NH}\right), 3.12\left(\mathrm{~s}, 2 \mathrm{H}, \mathrm{CH}_{2}-\mathrm{Cl}\right)$, 
2.87(d, 2H, $\left.\mathrm{CH}_{2}\right), 2.76(\mathrm{~m}, 1 \mathrm{H}, \mathrm{CH}-\mathrm{ph})$; Anal. Calcd. For $\mathrm{C}_{20} \mathrm{H}_{19} \mathrm{ClN}_{2} \mathrm{O}$ (338.8): C, $70.90 \% ; \mathrm{H}, 5.65 \% ; \mathrm{Cl}, 10.46 \%$ \% $\mathrm{N}, 8.27$ \%. Found: C, $70.65 \%$;, $5.72 \%$; Cl, $10.57 \%$; N, $8.39 \%$.

\section{Synthesis of 1,2-diamino-5-oxo-4,5-dihydro-1H-pyrrole-3-carbonitrile (13).}

To a solution of compound $12(3.38 \mathrm{~g}, 0.01 \mathrm{~mol})$ in $(20 \mathrm{ml})$ dimethylformamide reacted with malonitrile $(0.66 \mathrm{~g}, 0.01 \mathrm{~mol})$. The mixture was heated under reflux for $2 \mathrm{hrs}$, and then allowed to cool. The solid product was collected and recrystallized from DMF as deep brown crystal; yield: $62 \%$; mp $272^{\circ} \mathrm{C}$; IR $\left(\mathrm{KBrymax} \mathrm{cm}^{-1}\right): 3345-3280\left(\mathrm{NH}_{2}\right), 3156(\mathrm{NH}), 2929\left(\mathrm{CH}_{\text {aliph }}\right)$, 2859 $\left(\mathrm{CH}_{2}\right.$ aliph), 2211(CN), 1660(C=O), 1461 $(\mathrm{C}=\mathrm{N}) ;{ }^{1} \mathrm{H}-\mathrm{NMR}$ (DMSO-d 6 , $\left.\delta \mathrm{ppm}\right): 9.54\left(\mathrm{~s}, 1 \mathrm{H}, \mathrm{OH}\right.$, disappeared by $\left.\mathrm{D}_{2} \mathrm{O}\right)$, $5.31\left(\mathrm{~s}, 2 \mathrm{H}, \mathrm{NH}_{2}\right.$, disappeared by $\left.\mathrm{D}_{2} \mathrm{O}\right)$, 3.77(s, $\left.1 \mathrm{H}, \mathrm{CH}-\mathrm{C}=\mathrm{OH}\right)$; Anal. Calcd. For $\mathrm{C}_{5} \mathrm{H}_{6} \mathrm{~N}_{4} \mathrm{O}(138.1): \mathrm{C}, 43.48 \% ; \mathrm{H}, 4.38$ $\%$; N, $40.56 \%$. Found: C, $43.55 \%$; H, $4.49 \%$; N, $40.68 \%$.

\section{CONCLUSION}

The key intermediates ethyl-3,5-diphenylcyclohex-2-en-1-one-6-carboxylate (1) and 3,5 diphenylcyclohex-2-en-1- one (5) are used as effective synthons where it reacted with different reagents to afford pyrazole, chromen, ketoketene S,Sacetal, benzothiazole and Pyrrole derivatives. The new compounds were elucidated by chemical and spectroscopic data while some of them were tested for antibacterial effect and screened highly activity on Gram positive, and moderate to poor on Gram negative.

\section{REFERENCES}

[1] Dhar D.N., 1981. The Chemistry of Chalcones and Related Compounds, Wiley, New York, p 213.

[2] Torigoo T., Arisawa M., lloch S., Fujiu M., and Mayuyama H.B., 1983. Biophysical Research Communications, 112:833-842.

[3] Sukumaran K., and Kuttan R., 1991. Journal of Ethnopharmacology, 36:93-96.

[4] Padmavathi V., Reddy B.J.M., Balaiah A., Reddy K.V., and Reddy D.B., 2000. Molecules, 5:1281-1286.

[5] Senguttuvan S., and Nagarajan S., 2010. International Journal of Chemistry, 2: 108-112.

[6] Tanaka M., Nara F., Suzuki K., Hosoya T. and Ogita T., 1997. Journal of the American Chemical Society, 119: 78717872.

[7] Chong B.D., Ji Y.I., Oh S.S., Yang J.D., Baik W., and Koo S., 1997. J Org Chem, 62, 9323.

[8] Aurell M.J., Gavina P., and Mestres R., 1994. Tetrahedron. 50, 2571.

[9] Horspool W., Armesto D., and Horwood E., 1992. Organic Photochemistry: AComprehensive Treatment, (New York), 2265.

[10] Treves G.R., Hill K.L., and Dorschner K.P., 1966. US Patent, 3272992.

[11] Constantino M.G, Junior V.L and DaSilva G.J., 2002. Molcules, 7, 456.

[12] Li H., Mayekar A.N., Narayana B., Yathirajan H.S. and Harrison W.T.A., 2009. Acta Crystallographica, E65:01533.

[13] Jasinski J.P., Butcher R.J., Mayekar A.N., Yathirajan H,S, Narayana B. 2009. Journal of Chemical Crystallography, 39:157-162.

[14] Yathirajan H.S., Mayekar A.N., Narayana, B., Sarojini B.K., and Bolte M., 2007. Acta Crystallographica, E63:0540.

[15] Yathirajan H.S., Mayekar A.N., Sarojini B.K., Narayana B., and Bolte M., 2007. Acta Crystallographica, E63:01012.

[16] Yathirajan H S, Sarojini B.K., Bindya S., Narayana B., and Bolte M., 2006. Acta Crystallographica, E62:04046.

[17] Yathirajan H.S., Narayana B., Ashalatha B.V., Sarojini B.K. and Bolte M., 2006. Acta Crystallographica, E62:04440.

[18] Barbas III C.F., Lerner R.A., Zhong G. and List B., 2001. U S Patent, 6326176.

[19] Ying-Quan Y., Zhu C., Hai-Feng W., Xing-Kuan C., Hai-Feng C., Chang-Wu Z., Hua X., Peng L. and Cang Z., 2009. Chemistry European Journal, 48(15), 13295-13298.

[20] Sreevidya T.V., Narayana B. and Yathirajan H.S., 2009. Central European Journal of Chemistry, 8:174-181.

[21] Wan P.H., Andrei V.I. and Shannon S.S., J. Am. Chem. Soc., 2013, 135 (37), 13664-13667.

[22] Tianning D., Doris P. and Shannon S.S., J. Am. Chem. Soc., 2013, 135 (22), 8205-8212.

[23] Li H., Mayekar A.N., Narayana B., Yathirajan H.S. and Harrison W.T.A., 2009. Acta Crystallographica, E65:01186.

[24] Gao X.C., Cao H., Zhang L.Q., Zhang B.W., Cao Y. and Huang C.H. J., 1999. Mater. Chem., 9, 1077-1080.

[25] Omyma A.A., Ahmed M.E. and Asmaa M.K., 2013. Synthetic Comm., in press.

[26] Metwally M.A., Abdel-Galil E., Amer F.A., Abdallah A.M., 2012. American Journal of Organic Chemistry. 2(1): 28-34.

[27] Barry A.L., Philadeliphia P.A.,1976. USA, 8. 